\title{
Determinación del nivel de riesgo en la ejecución de obras públicas por contrata en la UNCP 2010 - 2017
}

\section{Determination of the level of risk in the execution of public works by contract in the UNCP during 2010-2017}

'Chumbimuni Macavilca, A.P.; Soto Ramos, A.N.

Universidad Nacional del Centro del Perú

Email: achumbimunim@uncp.edu.pe

\section{Resumen}

La investigación tuvo el objetivo de determinar las causas que originan los riesgos al ejecutar las obras públicas modalidad por contrato en la UNCP, analizando y contrastando las licitaciones públicas desarrollados durante 2010-2017, información extraída de las oficinas de logística, contaduría y obras e infraestructura de la entidad, además del portal de OSCE, CGR, MEF e Infobras.

Usando instrumentos normativos de la Contraloría General de la República, órgano rector regulador del Sistema Nacional de Control y del OSCE; disgregando cada proceso, se identificó que no existe una adecuada administración de riesgos en la ejecución de las obras publicas por contrata, lo que conlleva alto riesgo en: oportunidad al detectarse que el tiempo de demora es más del doble al previsto en los estudios definitivos de PIPs y, el valor referencial sobredimensionado excediendo en $15 \%$ como valor de culminación, información corroborada con liquidaciones técnica financiera y extraído del portal de las entidades reguladoras además del portal amigable del MEF.

Se concluye que, en nuestro país el sistema nacional de control es anacrónico, obsoleto y controversial; una puerta abierta al mal uso de los fondos públicos, por efectuar el control posterior y no un control preventivo y simultaneo que conlleve a mejorar la calidad del gasto público; el sistema de abastecimiento; responde a un andamiaje jurídico tradicional, la Ley y su reglamento tienen vacíos, conllevando a malas prácticas de autoridades y; funcionarios del Estado, abiertos a favorecer al contratista mediante solución de controversias, sea arbitraje o conciliaciones, generando perjuicio económico al Estado.

Palabras clave: nivel de riesgo, gasto público, obras públicas, procedimiento administrativo de adquisicición, entidad reguladora

\section{Abstract}

The research had the objective of finding causes underlying risks in the execution of the public contract wor$\mathrm{ks}$ at the University, analyzing and contrasting public tenders held during 2010-2017, information extracted from the offices of logistics, accounting and infrastructure works of the entity, as well as the OSCE, CGR, MEF and Infobras portal.

Using policy instruments of Contraloría General de la República (General Comptroller of the Republic) regulating governing body from the national control system and from the OSCE; disaggregation of each processidentified that there is no adequate risk management in executing public works by contracting, which entails high risk in: opportunity as detected that the delay time doubles the anticipated one in the definitive studies of PIPs, and the oversized reference value exceeds in fifteen percent as the culmination value, corroborated with financial technical liquidations and extracted from the portal of regulating entities as well as the friendly MEF portal.

The conclusion is that the national control system in our country is anachronistic, obsolete and controversial; it is also an open door to public funds mis-using, because it is a post controland not preventive and simultaneous control involving improvements in the quality of public expenditure, the supply system responds to a traditional legal scaffolding, the law and its reglament have lacks that lead to bad practices of authorities and state officials, open to favor the contractor through resolution of disputes, being arbitration or conciliation acts, generating economic damage to the State.

Keywords: level of risk, public expenditure, public works, administrative procurement procedure, regulatory entity 


\section{Introducción}

El presente trabajo de investigación, tuvo como finalidad evaluar y determinar los niveles de riesgos a presentarse durante la ejecución contractual de proyectos de inversión en sus diferentes modalidades, buscando optimizar la gestión pública para fortalecer las políticas de desconcentración de las entidades, dentro del periodo de estudio (ocho años 2010 a 2017). Para ello, se trabajó en estrecha coordinación con los funcionarios de las oficinas inmersas: de logística, administración financiera y obras e infraestructura, para lograr demostrar los principales objetivos planteados; asimismo, encuadrar a lo establecido en el fortalecimiento de la gestión administrativa legal en la entidad para mejorar la administración de los recursos públicos al ejecutar obras públicas al interior de la universidad.

Teniendo en cuenta este marco, en el desarrollo del proyecto, se trabajó extrayendo información de la base de datos del Órgano Supervisor de Contrataciones del Estado OSCE encontrándose, en su sistema electrónico, datos históricos valiosos sobre el procedimiento y antecedentes sobre gestión de las contrataciones estatales para ejecutar obras públicas durante los años investigados. Existió restricciones en la universidad para acceder a los expedientes de contratación, técnico, perfil y liquidaciones técnica financiera de cada proceso.

Habitualmente, las entidades estatales y, específicamente la UNCP, ejecutó siete licitaciones públicas en el periodo de estudio; además, realmente en la totalidad de los procesos ejecutados existió un nivel de riesgo significativo, debido a la presunción de malas prácticas de los actores, principalmente gestores, operadores y quienes toman decisiones en los procesos de contratación pública en sus tres etapas: actuaciones preparatorias, desarrollo del proceso y la ejecución contractual.

En ese contexto, la presente investigación constituye un instrumento de evaluación y consulta, permitiendo conocer, de manera ágil y sencilla, las diversas variables a evaluar para saber administrar una gestión de riesgos adecuadamente, buscando mitigar para no tener efectos negativos y conducir óptimamente la ejecución de un contrato para culminar una obra derivado de un proyecto de inversión pública de manera satisfactoria y sin poner en riesgo los intereses del Estado.

Toda vez que, en el desarrollo del trabajo, se utiliza dos instrumentos de control para identificar los riesgos en el país, la normado por CGR y el otro del OSCE, como órganos reguladores y rector de las contrataciones públicas, concluyéndose que existe perjuicio contra el Estado por ser inoportunos, sumado a que todas las licitaciones públicas exceden a los montos establecidos en el valor referencial en los estudios definitivos, propuesta económica del contratista y contrato suscrito por la universidad.
Cuando se analizó los instrumentos para una adecuada administración de riesgos, el cual, de utilizarse adecuadamente y de manera responsable, mitiga riesgos que puedan presentarse en su ejecución, implementando el control preventivo, concurrente y simultaneo, por medio del Sistema Nacional de Control, quien al efectúar el control posterior, no identifica oportunamente el alto riesgo, principalmente por deficiencias de control en las entidades. Las recomendaciones descritas contribuirán y permitirán efectuar un gasto público de calidad, contribuyendo al logro del bienestar de la universidad, con prestaciones oportunas a precio real y que consolide el proceso de desarrollo y bienestar de la comunidad universitaria.

\section{Materiales y métodos}

Identificado el problema de investigación, el mismo guarda relación a un estudio de carácter histórico descriptivo y explicativo, para lograr encontrar la vinculación entre variables, todas cuantificables de la muestra extraída; se analizó las Licitaciones Públicas para ejecución de obras públicas (proyectos de inversión) por contrata desarrollada en los años base 2010 y final 2017 en la UNCP.

El método usado descriptivamente que permitió específicamente y particularmente identificar hechos suscitados en el procedimiento de las convocatorias de licitaciones públicas con la finalidad de seleccionar postores para ejecutar obras en el periodo de estudio y poder describir el por qué son inoportunas, de baja calidad, inconclusas y con un valor de culminación por encima del estimado o referencial, analizándose la variable direccionamiento al diseñar los factores de evaluación.

Instrumento usado para explicar las causas por el cual existen distorsiones polémicas y escandalosas en las contrataciones públicas, cual es el problema para no generar perjuicio al Estado, por qué existe y cómo se debe actuar para evitar el direccionamiento de los procesos de licitación pública al momento de establecer los factores de evaluación para seleccionar la mejor propuesta, y disminuir los niveles de riesgo en la contratación y ejecución.

La población estuvo conformada por el total de procesos licitaciones públicas desarrolladas en la UNCP. Fueron siete en sus diferentes modalidades, en los ocho años de estudio para ejecutar proyectos de inversión pública.

La técnica de recolección de datos fue documental y la muestra estratificada conformó las siete LP y; analizado de los expedientes de contratación que obran en la UNCP corroborada con las páginas del portal de CGR, SEACE, MEF e INFOBRAS. 


\section{Resultados}

\section{Análisis y procesamiento de datos aplicando las} tablas de CGR

Para efectuar el análisis y procesar los datos de las siete obras ejecutadas en el periodo de estudio, se toma como modelo la ejecución de construcción del "Auditorio Post Grado de la UNCP” adjudicado mediante LP N ${ }^{\circ}$ 02-2014-CELP-UN$\mathrm{CP}$ bajo la modalidad de contrato a suma alzada y sistema llave en mano; se otorgó la buena pro al Consorcio del Centro, fuente de financiamiento Recursos Ordinarios y Determinados; con un valor referencial equivalente a $\mathrm{S} /$. 8'242,969.44, coincidente con la propuesta económica del contratista y monto equivalente al contrato.

En el proceso de ejecución contractual, evaluado el expediente de contratación, expediente técnico y la liquidación técnica financiera, la universidad aprueba diferentes adicionales y deductivos según resoluciones Nos. 770-R2018; 1190-R-2018; 1251-R-2018; y 1266-R-2018 por el importe de S/.717,188.65 debido a que mediante informes del supervisor de la obra del PIP remite a la entidad expedientes técnicos vinculantes al primigenio, adjuntando las copias del cuaderno de obra e informe o justificación del residente de obra, motivando el pronunciamiento del área técnica de la Oficina de Obras e Infraestructura de la UNCP, luego de una revisión y, por encontrarse conforme Ley, al cumplir las formalidades como: descripción de especificaciones técnicas, metrados, análisis de costo, insumos, presupuesto adicional y deductivo; que luego da lugar aplicar la fórmula polinómica, reajuste del cronograma de ejecución, cuadernos de obra, planos, entre otros, los cuales se adjuntan al expediente, entre otras formalidades, generando sobrecostos que sobredimensionan el valor de ejecución de acuerdo a lo establecido en el expediente original, producto de las nuevas partidas mayormente a precios unitarios. Ver Tabla 1, análisis determinación de riesgos según liquidación de obras.

Tabla 1

Determinación de riesgos según liquidaciones de obras en UNCP

\begin{tabular}{clll}
\hline \multicolumn{1}{c}{ Proceso } & \multicolumn{1}{c}{ Sub proceso } & \multicolumn{1}{c}{ Etapa } & \multicolumn{1}{c}{ Objetivo de la etapa } \\
\hline & & $\begin{array}{l}\text { E.1.1.1. Determinación del } \\
\text { valor referencial }\end{array}$ & $\begin{array}{l}\text { O.1.1.1. Valor referencial en } \\
\text { relación al expediente técnico }\end{array}$ \\
$\begin{array}{l}\text { P.1. Liquidaciones técnicas y } \\
\text { financieras de obras concluidas }\end{array}$ & S.1.1. Liquidación Financiera & $\begin{array}{l}\text { E.1.1.2. Expediente técnico ti- } \\
\text { S.1.2. Liquidación técnica }\end{array}$ & $\begin{array}{l}\text { O.1.1.2. Demoras en la eje- } \\
\text { cución de la obra }\end{array}$ \\
& E.1.1.3. Ejecución contractual de obra & $\begin{array}{l}\text { O.1.1.3. Ampliaciones de pla- } \\
\text { zo en el contrato de obra }\end{array}$ \\
\hline
\end{tabular}

En el análisis de la Contraloría (Tabla 2), se aprecia en resumen que esta contratación de ejecución de un PIP, generó una ampliación de plazo contractual de 407 días adicionales que sumado a lo establecido en el contrato habría insumido un total de 767 días para su culminación, el cual representa el $213 \%$ en función al periodo establecido para su culminación.

Tabla 2

Ampliaciones de plazo al ejecutar el PIP

\begin{tabular}{cccc}
\hline $\begin{array}{c}\text { Ampliación } \\
\text { de plazo }\end{array}$ & Días & $\begin{array}{c}\mathbf{N}^{\mathbf{0}} \\
\text { Resolución }\end{array}$ & $\begin{array}{c}\text { Fecha } \\
\text { ampliación }\end{array}$ \\
\hline 04 & 120 & Res. No $^{\circ}$ 00500-R-2016 & $10 / 05 / 2016$ \\
05 & 60 & Res. No 0770-R-2016 & $22 / 08 / 2016$ \\
06 & 62 & Res. No 0963-R-2016 & $06 / 01 / 2017$ \\
07 & 35 & Res. No 1240-R-2017 & $07 / 03 / 2017$ \\
08 & 75 & Res. No 1251-R-2017 & $17 / 02 / 2017$ \\
10 & 20 & Res. No 1190-R-2017 & $27 / 01 / 2017$ \\
11 & 35 & Res. No 1266-R-2017 & $16 / 03 / 2017$ \\
Total & $\mathbf{4 0 7}$ & & \\
\hline
\end{tabular}

El cual, como es lógico, ocasiona sobrecostos, malestar a los usuarios, perjuicio económico al Estado. Estando comprobado que se vulneró el sistema de contrataciones públicas por haberse ejecutado la prestación inoportunamente y en términos del valor referencial con un sobrecosto.

Continuando el análisis de los datos procesados en la Tabla 3, se describe y aprecia que para el caso de análisis existió un total de 191 días de ampliación de plazo, donde, como caso atípico, es necesario comentar que existió una ampliación de plazo por privacidad de libertad del contratista, el cual está al margen de los intereses de la institución y del Estado.

En las tablas 4 y 5 , se efectúa un análisis integral de la valoración de riesgo del proceso materia de análisis, identificando los controles, escala y determinación de controles clave; tal como se puede confirmar en el OSCE, para finalmente construir la matriz riesgo control en el cual se aprecia el objetivo, probabilidad, impacto, riesgo y nivel de eficacia y de riesgo residual. Según la escala de valoración de la Contraloría, confirma que en este PIP, en su proceso de ejecución, existió un nivel de riesgo significativo. 
Tabla 3

Motivo de ampliación de plazo

\begin{tabular}{|c|c|c|c|}
\hline $\mathbf{N}^{0}$ Resolución & Ampliación de plazo & Motivo & Días \\
\hline Resolución Nº 1483-R-2017 & $\mathrm{AP} \mathrm{N}^{\mathrm{o}} 01$ & Privación de la libertad, caso fortuito de fuerza mayor & 24 días \\
\hline Resolución Nº 1647-R-2017 & $\mathrm{AP} \mathrm{N}^{\circ} 02$ & $\begin{array}{l}\text { Aprobación de expediente técnico del adicional de la Obra } \\
\mathrm{N}^{\mathrm{o}} 02 \text {, deductivo de la Obra } \mathrm{N}^{\circ} 02 \text { y adicional deductivo } \\
\text { vinculante de Obra } \mathrm{N}^{\mathrm{o}} 02 \text {. }\end{array}$ & 45 días \\
\hline Resolución Nº 1781-R-2017 & $\mathrm{AP} \mathrm{N}^{\mathrm{o}} 03$ & $\begin{array}{l}\text { Demora de la entidad en absolución de consultas sobre eje- } \\
\text { cución de adicionales y deductivos de la Obra } \mathrm{N}^{\mathrm{o}} 01\end{array}$ & 15 días \\
\hline Resolución Nº 1866-R-2017 & $\begin{array}{l}\text { Suspensión de plazo } \\
\text { ejecución de obra }\end{array}$ & $\begin{array}{l}\text { Aprobación de la ejecución del presupuesto adicional-de- } \\
\text { ductivo de Obra } \mathrm{N}^{\mathrm{o}} 03 \text {. }\end{array}$ & 60 días \\
\hline Resolución Nº 1947-R-2017 & $\mathrm{AP} \mathrm{N}^{\mathrm{o}} 04$ & $\begin{array}{l}\text { Aprobación de la ejecución del presupuesto adicional-de- } \\
\text { ductivo de Obra } \mathrm{N}^{\circ} 04\end{array}$ & 30 días \\
\hline Resolución Nº 2138-R-2017 & $\mathrm{AP} \mathrm{N}^{\circ} 05$ & Por la ejecución de mayores medrados. & 10 días \\
\hline \multirow[t]{2}{*}{ Resolución Nº 2205-R-2017 } & $\mathrm{AP} \mathrm{N}^{\circ} 06$ & $\begin{array}{l}\text { Aprobación de la ejecución del presupuesto adicional de- } \\
\text { ductivo de Obra } \mathrm{N}^{\circ} 05\end{array}$ & 15 días \\
\hline & & Total & 191 días \\
\hline
\end{tabular}

Tabla 4

Determinación de controles claves

\begin{tabular}{|c|c|c|c|c|c|c|c|c|c|}
\hline \multirow[b]{2}{*}{ Riesgo } & \multirow[b]{2}{*}{ Control } & \multicolumn{5}{|c|}{$\begin{array}{c}\text { Cumplimiento de las NCI } \\
\text { Actividades de control (valoración) }\end{array}$} & \multirow{2}{*}{$\begin{array}{c}\text { Valor } \\
\text { promedio de } \\
\text { la suma (a,b,- } \\
\text { c,d,e) }\end{array}$} & \multicolumn{2}{|c|}{$\begin{array}{l}\text { Importancia del control } \\
\text { sobre los riesgos }\end{array}$} \\
\hline & & 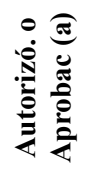 & 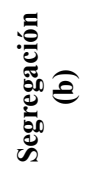 & 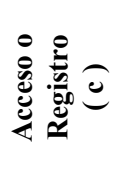 & 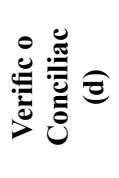 & 总 & & $\begin{array}{c}\text { Clave o } \\
\text { fundamental }\end{array}$ & Prescindible \\
\hline R.1.1.1.1. & C.1.1.1.1. & 3 & & & 3 & 3 & 3 & $X$ & \\
\hline R.1.1.1.2. & C.1.1.1.2. & & 2 & 2 & 3 & 3 & 2.5 & $X$ & \\
\hline R.1.1.2.1. & C.1.1.2.1. & 3 & 2 & & & 2 & 2.33 & $X$ & \\
\hline R.1.1.2.2. & C.1.1.2.2. & 2 & 2 & & 1 & & 1.66 & $X$ & \\
\hline R.1.1.3.1. & C.1.1.3.1. & 3 & & 3 & & 2 & 2.66 & $X$ & \\
\hline R.1.1.3.2. & C.1.1.3.2. & & 2 & & 2 & 2 & 2 & $X$ & \\
\hline
\end{tabular}

Tabla 5

Matriz riesgo - control

\begin{tabular}{lcc}
\hline \multicolumn{1}{c}{$\begin{array}{c}\text { Indicador del } \\
\text { riesgo residual }\end{array}$} & Valor & $\begin{array}{c}\text { Nivel de } \\
\text { riesgo residual }\end{array}$ \\
\hline Nivel del riesgo & $2.40 \mathrm{~A} 9.00$ & No aceptable \\
& $1: 00 \mathrm{~A} 2.39$ & Importante \\
& $8.80 \mathrm{~A} 0.99$ & Moderado \\
$\begin{array}{l}\text { Nivel de eficiencia } \\
\text { del control }\end{array}$ & $0.60 \mathrm{~A} 0.79$ & Tolerante \\
& $0.10 \mathrm{~A} 0.59$ & Aceptable \\
\hline
\end{tabular}

De usarse este instrumento durante su ejecución de manera permanente, nos mostraría los indicios de (LINARES JARA) una real valoración de riegos, y que le mismo permitiría realizar los correc- tivos implementados de manera concurrente, con el cual se tendría una gestión de riesgos bastante prudente, conllevando a la ejecución y culminación de un proyecto de inversión por contrata en forma oportuna, de la mejor calidad y a un valor prudente.

Análisis y procesamiento de datos con instrumentos del OSCE

Como instrumento adicional de análisis, se tomó la Resolución $\mathrm{N}^{\circ}$ 284-2017-OSCE/PRE y Directiva $\mathrm{N}^{\circ}$ 012-2017-OSCE/CD, por el cual, con la finalidad que las entidades tengan un instrumento integral de la gestión de riesgos al ejecutar los PIPs, el cual en cuatro procesos permite: identificar, analizar, planificar la respuesta y asignar riesgos. 
El cual, en el tiempo devino en controversial por su inaplicación al interior de las entidades, debido a los escándalos de la mayor parte de los procesos de ejecución de obras públicas o proyectos de inversión por contrata. Significando un contexto bastante disconforme que genera desconfianza de todos los actores involucrados, principalmente los beneficiarios o población meta, habiéndose confirmado los niveles de negociación y malas prácticas.

Por ello, que de manera referencial y como instrumento adicional de análisis, se aplicó y efectuó un somero análisis que permita enriquecer el trabajo de investigación, de cómo algunos instrumentos, principalmente de control y evaluación, solo quedan en el tintero y no son usados de la manera más adecuada; de ahí deviene la desconfianza que tiene el país en conjunto de nuestro Sistema Nacional de Control a cargo de la Contraloría General de la República, dado que a su vista y paciencia viene ocurriendo las grandes deformaciones en las contrataciones públicas en nuestro país y, desarrollado a todo nivel, incumpliéndose con que los recursos del Estado deben usarse de manera prudente racional y responsable debido a que son escasos.

La investigación plantea como principal interrogante el ¿cómo se implementa la gestión de riesgos en la planificación de la ejecución de obras con los instrumentos del OSCE?

Con la entrada en vigencia de las modificaciones a la Ley de Contrataciones del Estado, y su Reglamento, en los contratos de obra se deben identificar y asignar los riesgos previsibles de ocurrir durante su ejecución.

Este análisis es parte del expediente, la implementación de la gestión de riesgos busca incrementar la eficiencia de las inversiones en las obras públicas. Buscando facilitar la aplicación, OSCE publicó la Directiva $\mathrm{N}^{\circ} 012$ 2017-OSCE/CD, con el cual, el enfoque integral de gestión de riesgos abarca cuatro procesos detallados en el párrafo anterior.

A fin de mejorar el análisis y aplicación de la gestión de riesgos, a continuación, se aplicó los cuatro pasos en la Tabla 6, según establece la guía práctica de la Contraloría para cada proceso de la gestión de riesgos.

Tabla 6

Aplicación de gestión de riesgos según OSCE.

Paso 1 Identificar riesgos En este proceso se deben identificar los riesgos previsibles de ocurrir durante la ejecución de la obra En este proceso se analiza la probabilidad de ocurrencia del riesgo y el impacto que tendría en la ejecución de la obra, clasificando los riesgos identificados en función a su prioridad sea esta alto, media-

Paso 2 Analizar riesgos
no o bajo. Para ello, la directiva establece que la entidad puede usar la matriz de probabilidad e im-
pacto contenida en la Guía PMBOK del PMI ${ }^{\circ}$ o, caso contrario, desarrollar su propia metodología.

Paso 2 Analizar riesgos
no o bajo. Para ello, la directiva establece que la entidad puede usar la matriz de probabilidad e im-
pacto contenida en la Guía PMBOK del PMI ${ }^{\circ}$ o, caso contrario, desarrollar su propia metodología.

En este proceso se selecciona la estrategia y acciones a seguir para dar respuesta al riesgo identificado. Asimismo, se identifica el disparador de riesgo; es decir, la situación que alertará de la presencia del riesgo. Las estrategias que se pueden adoptar son las siguientes, conforme a la Guía del PMBOK del PMI ${ }^{\circledR}$ :

- Mitigar, que implica llevar a cabo acciones que permitan reducir la probabilidad de ocurrencia o el impacto de un riesgo sobre la obra.

Planificar la

Paso 3 res-puesta a riesgos
- Evitar, que supone eliminar la(s) causa(s) generadoras del riesgo o proteger al proyecto del impacto del riesgo.

- Esta estrategia puede generar la modificación de las condiciones iníciales del proyecto.

- Aceptar, que implica reconocerla existencia del riesgo y determinar, de ser el caso, las medidas a adoptar si el riesgo se materializa.

- Transferir, que supone trasladar el impacto negativo del riesgo y la responsabilidad de gestionar adecuadamente el mismo, a un tercero. Por ejemplo, a través de la contratación de un seguro.

Finalmente, se debe asignar el riesgo a la parte que está en mejor capacidad para controlarlo. Principales riesgos en la ejecución de obras, deficiencias en el diseño del proyecto de pre inversión, estudio definitivo deficiente, deficiencias en las labores administrativas, muchas veces desinterés de los beneficiarios, deficiencia en la asignación de recursos presupuestales, demoras, falta de personal capacitado en la unidad ejecutora, deficiente calidad de los equipos/insumo y materiales, problemas climatológicos y/o físico geográficos, deficiente desempeño de los contratistas/consultores, limitaciones en el marco legal, deficiencias en los arreglos institucionales, aspectos administrativos, modalidad de ejecución inapropiada.
Este instrumento de análisis, bastante importante para la ejecución de obras públicas por contrata a nivel de PIPs u otros procesos que se desarrolla al interior de las entidades, es recomendable su uso e implementación, toda vez que permite una evaluación permanente y constante para esa forma evitar posterior inconveniente como se viene presentado y ocurre constantemente en todas las entidades del Estado.

Igualmente, en la Tabla 7, para complementar nuestro análisis de evaluación de riesgos, planteamos otro instrumento de análisis que permite identificar los riesgos a presentarse dentro de la ejecución de un PIPs al interior de las entidades. 
Tabla 7

Evaluación de la gestión de riesgos

\begin{tabular}{|c|c|c|}
\hline $\mathbf{N}^{\mathbf{o}}$ & Interrogante & Descripción \\
\hline 1 & $\begin{array}{l}\text { ¿La modalidad de } \\
\text { ejecución fue la } \\
\text { adecuada? }\end{array}$ & $\begin{array}{l}\text { En el análisis de la investigación, se tiene claro que en suma alzada es un monto tope, en la cual se } \\
\text { debe incluir todo aquello que contiene el expediente técnico (Salinas, 2009) y las bases de concur- } \\
\text { so para el proyecto. Un contrato a suma alzada, generalmente es elaborado por la entidad, el con- } \\
\text { tratista es el que asume todo el riesgo por el diseño, toda vez que si se identifican errores de diseño } \\
\text { llevan a sobre costos en la ejecución de la obra, por diferentes motivos, como: incompatibilidades, } \\
\text { falta de información o puede ser especificaciones técnicas erróneas. Estos errores son considerados } \\
\text { como adicionales de obra, algunas veces asume la entidad, cuando debe asumir el contratista; asi- } \\
\text { mismo, el contratista asume el riesgo de su propio mitrado, ya que, una vez adjudicado el proyecto, } \\
\text { no puede modificar las cantidades estimadas. }\end{array}$ \\
\hline
\end{tabular}

Mientras más detallado sea el diseño (planos), mejor será la cesión de riesgos de la entidad al contratis-

2 ¿El diseño fue adecuado? quien estara más abierto a aceptar dicho riesgo por a calidad del diseño; es decir, el hacer una alta inversión en la etapa de planeamiento puede llevar a optimizar precios considerablemente durante la construcción de la obra (Salinas, 2009).

En el análisis de la investigación, de conformidad al organismo supervisor de las contrataciones del Estado. Ley de Contrataciones y su reglamento comunicados, no hubo modalidad de contratación de precios unitarios cuando se tiene metrados variables, pero los precios unitarios son

¿El número excesi- fijos, puede darse el caso cuando la obra no tenga partidas muy complejas y las cantidades sean difícil de estimar, estas modalidades se dan generalmente en carreras, túneles o movimientos de dificulta la ejecución? tierra cuando las condiciones del suelo pueden o no coincidir con el estudio de suelos. En un contrato bajo esta modalidad el riesgo del metrado se traslada a la entidad, mientras que el riesgo del costo de materiales y eficiencia al contratista. Este contrato puede ser justo y, para ello, se deben conocer las partidas involucradas y tener sus análisis de precios unitarios bien definidos, caso contrario puede ser muy complicado de controlar.

¿Las metas fueron Las metas son realistas, debidamente aprobadas y autorizadas para su ejecución
realistas?

¿La estrategia de No, porque en todas las obras no se cumplieron con las especificaciones técnicas, hubo muchos ries-

5 ejecución fue efi- gos de ampliaciones de plazo en la ejecución, los montos contractuales han sido significativamente ciente? incrementados debidos a adicionales de obras y ampliaciones de plazos a cuenta de la entidad.

¿Los beneficiarios

6 asumieron compro- Se asume compromisos cuando termina la obra es recibida y puesta en funcionamiento. misos reales?

Es notorio el poco interés de los funcionarios y operadores de las contrataciones (órgano encargado de las contrataciones - OEC) en implementar estos instrumentos de bastante relevancia con la finalidad principalmente que no exista disyuntivas en la ejecución de las contrataciones públicas, de exceso de ampliaciones, obras inconclusas que conlleva luego al tradicional arbitraje donde siempre el estado sale perdiendo.

Está demostrado, que con todos estos instrumentos, se pondría a buen recaudo los intereses del Estado, evitando con ello sobrecostos y principalmente puedan ejecutarse oportunamente dentro de los plazos, de esta forma evitar el perjuicio que se ocasiona a los beneficiarios o usuarios.

\section{Discusión}

Discusión resultados de los procesos evaluados

Efectuado la discusión de resultados de las seis licitaciones públicas, evaluadas en el desarrollo de la presente investigación, se tienen como resultados aspectos bastante controversiales que motivan a dar una apreciación de ciertos indicios de actuaciones bajo el contexto de las malas prácticas (Contraloría General de la República del Perú), al poner en riesgo los interés de la entidad y del Estado con un sobredimensionamiento excesivo en dos aspectos: inoportunidad en el periodo de ejecución en función a lo programado en los estudios tanto preliminar y definitivo, como viene a ser el perfil y expediente técnico, valor referencial y de culminación, y la valoración de riesgo para confirmar los resultados obtenidos.

En la Tabla 8, se efectuó analíticamente la discusión del resultado de una de las variables importante que viene a ser "valor referencial vs valor de culminación”, en el cual, los seis PIPs insumió al Estado un valor adicional de S/.4'390,468.40 para su culminación, la misma serie el monto en perjuicio del Estado, toda vez que se debe culminar al costo establecido como valor referencial y monto con el cual se suscribió el contrato. 
Tabla 8

Cuadro discusión de resultado valor referencial y valor de culminación de la obra

\begin{tabular}{|c|c|c|c|c|c|c|}
\hline $\mathbf{N}^{\mathbf{0}}$ & Obra & Adjudicación & $\begin{array}{l}\text { Modalidad de } \\
\text { contratación }\end{array}$ & Valor contrato & $\begin{array}{l}\text { Valor total } \\
\text { obra }\end{array}$ & $\begin{array}{l}\text { Ampliaciones } \\
\text { y deducciones }\end{array}$ \\
\hline 1 & $\begin{array}{l}\text { Instalación de auditorio de la Uni- } \\
\text { versidad Nacional del Centro del } \\
\text { Perú }\end{array}$ & LP. No 002-2014-CELP & $\begin{array}{l}\text { Suma alzada- } \\
\text { llave en mano }\end{array}$ & $8.242 .969,44$ & $9.600 .767,33$ & $1.357 .797,89$ \\
\hline 2 & $\begin{array}{l}\text { Construcción y equipamiento de } \\
\text { los laboratorios de química analíti- } \\
\text { ca en la planta piloto metalúrgico } \\
\text { de Yauris de la Facultad de Meta- } \\
\text { lurgia y de Materiales de la UNCP. }\end{array}$ & $\begin{array}{l}\text { L.P. No001-2014-CELP- } \\
\text { UNCP }\end{array}$ & $\begin{array}{l}\text { A suma alzada } \\
\text { llave en mano }\end{array}$ & $2.121 .884,46$ & $2.340 .081,53$ & $218.197,07$ \\
\hline 3 & $\begin{array}{l}\text { Creación de aulas y servicios } \\
\text { higiénicos de la Facultad de Cien- } \\
\text { cias Agrarias de la UNCP - Río Ne- } \\
\text { gro Satipo Junín }\end{array}$ & $\begin{array}{l}\text { AS-SM-2-2016-CSASO- } \\
\text { UNCP-1 }\end{array}$ & A suma alzada & $1.468 .948,00$ & $1.596 .685,27$ & $127.737,27$ \\
\hline 4 & $\begin{array}{l}\text { Mejoramiento de los residuos sóli- } \\
\text { dos de la ciudad universitaria de la } \\
\text { UNCP. }\end{array}$ & $\begin{array}{l}\text { OBRAS POR IMPUES- } \\
\text { TO }\end{array}$ & Por contrata & $482.572,98$ & $569.851,30$ & $87.278,32$ \\
\hline 5 & $\begin{array}{l}\text { Construcción y equipamiento del } \\
\text { pabellón de laboratorios de inves- } \\
\text { tigación científica y tecnológica de } \\
\text { ciencias agrarias de la UNCP El } \\
\text { Mantaro UNCP Hyo Junín }\end{array}$ & $\begin{array}{l}\text { LP N }^{\circ} 001-2011-\mathrm{UN}- \\
\text { CP-EO }\end{array}$ & A suma alzada & $4.258 .968,63$ & $4.657 .117,57$ & $398.148,94$ \\
\hline 6 & $\begin{array}{l}\text { Construcción y equipamiento del } \\
\text { pabellón de laboratorios de inves- } \\
\text { tigación científica y tecnológica } \\
\text { de las facultades de arquitectura, } \\
\text { ingeniería civil, minas, mecánica, } \\
\text { metalúrgica y sistemas de la UNCP }\end{array}$ & $\begin{array}{l}\text { L.P. } \mathrm{N}^{\circ} 001-2010-\mathrm{UN}- \\
\text { CP-EO }\end{array}$ & A suma alzada & $6.718 .014,56$ & $8.447 .486,72$ & $1.729 .472,16$ \\
\hline 7 & $\begin{array}{l}\text { Construcción e implementación de } \\
\text { planta piloto en investigación e in- } \\
\text { novación de la FAIIA de la UNCP } \\
\text { El Mantaro }\end{array}$ & $\begin{array}{l}\text { AS N }{ }^{\circ} 001-2017-C O S L- \\
\text { PO-UNCP DERIVADADE } \\
\text { LALP N }{ }^{\circ} 001-2016-C O S L- \\
\text { PO-UNCP }\end{array}$ & $\begin{array}{l}\text { A suma alzada } \\
\text { llave en mano }\end{array}$ & 6'819,639.79 & $7^{\prime} 291,476.54$ & $471,836.75$ \\
\hline \multicolumn{3}{|c|}{ Totales } & & 30'112,997.86 & & 4’390,468.40 \\
\hline
\end{tabular}

La Ley de Contrataciones y su reglamento, menciona que es una puerta abierta a las malas prácticas de los actores al interior de las entidades, y analizando, coincidentemente este valor representa en promedio montos menor o igual al $15 \%$, el cual se encuentra respaldado con el andamiaje normativo que para este caso funciona correctamente

En la Tabla 9, discusión de resultados, de manera comparativa del periodo de ejecución de la obra, evaluando el periodo establecido en los estudios y el contrato en función al establecido en la liquidación técnica establecido como causas (variables de X1 al X6), tiene el mismo comportamiento, exceden en demasía, el más alto es de 450 y el menor de 34 días y, en conjunto, para los seis PIPs evaluados se tiene que insumió 1,536 días adicionales, generando perjuicio en este caso a los estudiantes.

El promedio de los seis PIPs es de 256 días, apreciándose que el que insumió mayor tiempo fue la "Construcción y equipamiento del pabellón de laboratorios de investigación científica y tecnológica de las facultades de arquitectura, ingeniería civil, minas, mecánica, metalúrgica y sistemas de la UNCP, con un exceso de 450 días adicionales de demora en función al previsto.

Confirmando lo señalado en la hipótesis, que no es solo coincidencia que existe demasiada demora en las prestaciones de ejecución de los proyectos de inversión por contrata y, ante una deformación del sistema normativo de las contrataciones públicas en el país es una puerta abierta para que los empresarios actúen incorrectamente, incluso con indicios de malas prácticas y negociaciones, debilitando el uso adecuado de los recursos estatales, e incumplimiento en atender las necesidades de estudiantes. Además, la variable efecto (compuesto de Y1 a Y3) confirma lo señalado

\section{Discusión nivel y valoración de riesgos}

Conforme a lo precisado en el marco teórico del presente trabajo de investigación, se tomó en consideración aplicar los procedimientos establecidos para establecer una gestión de riesgos de manera prudente y que en las entidades existe un contexto bastante diferenciado en 
Tabla 9

Discusión de resultado comparativo, tiempo de ejecución de obra

\begin{tabular}{|c|c|c|c|c|c|c|c|c|c|}
\hline \multirow[b]{2}{*}{$\mathbf{N}^{\circ}$} & \multicolumn{6}{|c|}{ Causas } & \multicolumn{3}{|c|}{ Efecto } \\
\hline & $\begin{array}{c}\text { X1 } \\
\text { Estudio } \\
\text { técnico mal } \\
\text { elaborados }\end{array}$ & $\begin{array}{c}\mathrm{X2} \\
\text { Descono- } \\
\text { cimiento de } \\
\text { la normativa } \\
\end{array}$ & $\begin{array}{c}\text { X3 } \\
\text { Deficiente } \\
\text { asignación } \\
\text { presupuesta }\end{array}$ & $\begin{array}{c}\mathrm{X} 4 \\
\text { Malas } \\
\text { prácticas de } \\
\text { operadores } \\
\text { logísticos } \\
\end{array}$ & $\begin{array}{c}\text { X5 } \\
\text { Atribuible } \\
\text { al } \\
\text { contratista }\end{array}$ & $\begin{array}{c}\text { X6 Con- } \\
\text { troversias } \\
\text { por vacios } \\
\text { legales. }\end{array}$ & $\begin{array}{c}\text { Y1 } \\
\text { Ampliaciones } \\
\text { presupuestales }\end{array}$ & $\begin{array}{c}\text { Y2 } \\
\text { Ampliaciones } \\
\text { de plazo }\end{array}$ & $\begin{array}{c}\text { Y3 } \\
\text { Nivel de } \\
\text { riesgo }\end{array}$ \\
\hline 1 & & & & $X$ & & $\mathrm{X}$ & $1,357,797.00$ & 404 días & ALTO \\
\hline 2 & $\mathrm{X}$ & $\mathrm{X}$ & & $\mathrm{X}$ & & & $218,197.07$ & 234 días & ALTO \\
\hline 3 & & & $\mathrm{X}$ & & & & $127,737.27$ & 56 días & BAJO \\
\hline 4 & & & $\mathrm{X}$ & & $\mathrm{X}$ & & $87,278.32$ & 34 días & BAJO \\
\hline 5 & & & & $X$ & & & $398,148.94$ & 178 días & MEDIO \\
\hline 6 & & & $\mathrm{X}$ & $X$ & & & $1,729,472.16$ & 450 días & ALTO \\
\hline 7 & & & $X$ & & & $X$ & $471,836.75$ & 109 días & MEDIO \\
\hline & & & Totale & & & & $\mathrm{S} / . \mathbf{4 , 3 9 0 , 4 6 8 . 4 0}$ & 1,536 días & \\
\hline
\end{tabular}

establecer sistemas de control que conlleven a mitigar los tipos de riesgos que enfrentan.

Más aún, se comprobó que no existe la voluntad política de las autoridades, menos el compromiso de los operadores logísticos (OEC, área usuaria y técnica) al interior de las entidades para implementar los instrumentos precisados en el presente trabajo (porque todos ellos se encuentran al alcance de cualquier ciudadano) y, que ello conllevaría a tener una modalidad de control preventivo, detectivo y correctivo que permita poner a buen recaudo los escasos recursos estatales y contrarrestar los riesgos que se presentan al ejecutar el gasto público en la ejecución de obras públicas.

Por ello, en este acápite se desarrolla la discusión de resultados, habiendo aplicado toda la información recopilada del portal del OSCE, de la Oficina de Abastecimientos Servicios Generales, Obras e Infraestructura y, Contaduría y Administración Financiera de donde se extrajo información relacionado a las licitaciones públicas desarrolladas en el periodo de estudio y, aplicado los siete PIPs materia de investigación, los resultados que a continuación se describe.

En la Tabla 10, luego de haber efectuado el respectivo análisis de los resultados obtenidos se identificó los riesgos, fuente, causa, efecto y valoración del riesgo de los siete procesos ejecutados en el periodo de estudio, el cual llevó a determinar y discutir el nivel de investigación a ponderar la valoración para su interpretación posterior.

En la Tabla 11, se muestra la valoración del riesgo, efectuado el gráfico $\mathrm{N}^{\circ} 01$, la ponderación de la valoración del riesgo resulta alta como común denominador y segunda opción mediana, y tercera ponderación como baja una sola obra ejecutada en la modalidad de obra por impuestos, en el cual, por su modalidad de ejecución donde no existe mucha injerencia de los operadores logísticos, se puede observar que el nivel de riesgo es menor. Curiosamente, en el proceso señalado, se habría aminorado el nivel de riesgo, porque administrativamente la universidad no efectuó el pago de la prestación, sino fue por medio de Sunat, mediante notas de crédito negociables.

En la Tabla 12 y Figura 1, se tiene la discusión de resultados sobre valoración de riesgo, el cual confirma lo advertido anteriormente, en donde el nivel de riesgo es alto y mediano, cuanta más intervención existe de los operadores logísticos, hablando de las aéreas de logística, contaduría administración financiera, obras e infraestructura, área usuaria y técnica. Con ello, se estaría confirmando que la deformación de las contrataciones públicas es debido a los operadores, el sistema y la normatividad aún de ser pesada tiene muchas bondades de transparencia y equidad. Existe un nivel de riesgo alto en la normatividad por el mal uso interpretativo, donde se aduce una cantidad de vacíos que conlleva a que el sistema de solución de controversia en las contrataciones públicas sea tan desfavorable para el Estado por las malas prácticas en su interior de las personas que toman decisiones y tienen injerencia directa.

$\mathrm{Al}$ analizar por cada etapa, se tiene la fase de actos preparatorios al formular el expediente de contratación, donde el requerimiento de lo que se ejecutará (solicitud de ejecución de obra por contrata), la actividad homologación, estudio o indagación del mercado, valor referencial o estimado, determinación de la modalidad de contratación, certificación presupuestal, declaratoria de viabilidad para ejecución de proyectos de inversión pública, entre otros. (Salinas, 2011) 
Tabla 10

Identificación de riesgos, fuente, causa, efecto y valoración del riesgo.

\begin{tabular}{|c|c|c|c|c|c|c|c|c|c|c|}
\hline \multirow[b]{2}{*}{$\mathbf{N}$} & \multirow[b]{2}{*}{ Obra } & \multirow[b]{2}{*}{ Riesgos } & \multicolumn{2}{|c|}{ Fuente } & \multirow[b]{2}{*}{ Causas } & \multirow[b]{2}{*}{ Efectos } & \multirow[b]{2}{*}{ Prob } & \multirow[b]{2}{*}{ Imp } & \multirow{2}{*}{ 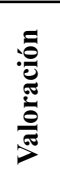 } & \multirow[b]{2}{*}{$\begin{array}{l}\text { Valor } \\
\text { riesgo }\end{array}$} \\
\hline & & & 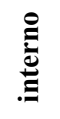 & 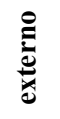 & & & & & & \\
\hline 1 & $\begin{array}{l}\text { Instalación de audi- } \\
\text { torio de la Univer- } \\
\text { sidad Nacional del } \\
\text { Centro del Perú }\end{array}$ & $\begin{array}{l}\text { a) Inadecuada } \\
\text { determinación del } \\
\text { VR. } \\
\text { b) Ampliaciones } \\
\text { de plazo }\end{array}$ & X & & $\begin{array}{l}\text { Desconocimiento } \\
\text { de la normativa } \\
\text { Atrasos y/o pa- } \\
\text { ralizaciones por } \\
\text { causas no atribui- } \\
\text { bles al contratista }\end{array}$ & $\begin{array}{l}\text { Mayores costos } \\
\text { directos } \\
\text { Inoportuna aten- } \\
\text { ción a los usuarios }\end{array}$ & 3 & 3 & 9 & Alta \\
\hline 2 & $\begin{array}{l}\text { Construcción y } \\
\text { equipamiento de } \\
\text { los laboratorios de } \\
\text { química analítica } \\
\text { en la Planta Piloto } \\
\text { Metalúrgico de } \\
\text { Yauris de la Facul- } \\
\text { tad de Metalurgia y } \\
\text { de Materiales de la } \\
\text { UNCP. }\end{array}$ & $\begin{array}{l}\text { a) Inadecuada } \\
\text { determinación del } \\
\text { Valor Referencial } \\
\text { b) Ampliaciones } \\
\text { de plazo }\end{array}$ & $X$ & $X$ & $\begin{array}{l}\text { Valores referen- } \\
\text { ciales sobre esti- } \\
\text { mados } \\
\text { Demora de la } \\
\text { entidad en absolu- } \\
\text { ción de consultas }\end{array}$ & $\begin{array}{l}\begin{array}{l}\text { Adicionales de } \\
\text { obras }\end{array} \\
\text { Modifica la ruta } \\
\text { crítica de la ejecu- } \\
\text { ción de obra }\end{array}$ & 3 & 3 & 9 & Alta \\
\hline 3 & $\begin{array}{l}\text { Creación de aulas y } \\
\text { servicios higiénicos } \\
\text { de la Facultad de } \\
\text { Ciencias Agrarias } \\
\text { de la UNCP - Rio } \\
\text { Negro Satipo Junín }\end{array}$ & $\begin{array}{l}\text { a) Inadecuada } \\
\text { determinación del } \\
\text { Valor Referencial } \\
\text { b) Ampliaciones } \\
\text { de plazo }\end{array}$ & $X$ & $X$ & $\begin{array}{l}\text { Valores referen- } \\
\text { ciales incompletos } \\
\text { Ejecución de ma- } \\
\text { yores metrados }\end{array}$ & $\begin{array}{l}\text { Adicionales de } \\
\text { obras } \\
\text { Modifica la ruta } \\
\text { crítica de la ejecu- } \\
\text { ción de obra }\end{array}$ & 3 & 2 & 6 & Mediana \\
\hline 4 & $\begin{array}{l}\text { Mejoramiento de } \\
\text { los residuos sólidos } \\
\text { de la ciudad univer- } \\
\text { sitaria de la UNCP. }\end{array}$ & $\begin{array}{l}\text { a) Inadecuada } \\
\text { determinación del } \\
\text { Valor Referencial } \\
\text { b) Ampliaciones } \\
\text { de plazo }\end{array}$ & $X$ & $\mathrm{X}$ & $\begin{array}{l}\text { Valores referencia- } \\
\text { les sin sustento } \\
\text { Aprobación de la } \\
\text { ejecución del Presu- } \\
\text { puesto adicional. }\end{array}$ & $\begin{array}{l}\text { Adicionales de } \\
\text { obras } \\
\text { Modifica la ruta } \\
\text { crítica de la ejecu- } \\
\text { ción de obra }\end{array}$ & 1 & 3 & 3 & Mediana \\
\hline 5 & $\begin{array}{l}\text { Construcción y } \\
\text { equipamiento del } \\
\text { pabellón de labo- } \\
\text { ratorios de inves- } \\
\text { tigación científica } \\
\text { y tecnológica de } \\
\text { ciencias agrarias de } \\
\text { la UNCP El Manta- } \\
\text { ro UNCP Hyo Junín }\end{array}$ & $\begin{array}{l}\text { a) Inadecuada } \\
\text { determinación del } \\
\text { Valor Referencial }\end{array}$ & $X$ & $\mathrm{X}$ & $\begin{array}{l}\text { Valores referen- } \\
\text { ciales sobre esti- } \\
\text { mados } \\
\text { Demora de la enti- } \\
\text { dad en absolución } \\
\text { de consultas sobre } \\
\text { ejecución de adi- } \\
\text { cionales y deducti- } \\
\text { vos de la obra }\end{array}$ & $\begin{array}{l}\text { Modifica la ruta } \\
\text { crítica de la eje- } \\
\text { cución de obra }\end{array}$ & 3 & 2 & 6 & Mediana \\
\hline 6 & $\begin{array}{l}\text { Construcción y } \\
\text { equipamiento del } \\
\text { pabellón de labo- } \\
\text { ratorios de inves- } \\
\text { tigación científica } \\
\text { y tecnológica de } \\
\text { las facultades de } \\
\text { arquitectura, inge- } \\
\text { niería civil, minas, } \\
\text { mecánica, metalúr- } \\
\text { gica y sistemas de } \\
\text { la UNCP }\end{array}$ & $\begin{array}{l}\text { a) Inadecuada } \\
\text { determinación del } \\
\text { Valor Referencial }\end{array}$ & $\mathrm{X}$ & $X$ & $\begin{array}{l}\text { Valores referen- } \\
\text { ciales incomple- } \\
\text { tos (sin considerar } \\
\text { IGV) } \\
\text { Aprobación de } \\
\text { la ejecución del } \\
\text { presupuesto adi- } \\
\text { cional - Deducti- } \\
\text { vo } N^{\circ} 05\end{array}$ & $\begin{array}{l}\text { Modifica la ruta } \\
\text { crítica de la eje- } \\
\text { cución de obra }\end{array}$ & 2 & 2 & 4 & Bajo \\
\hline 7 & $\begin{array}{l}\text { Construcción e im- } \\
\text { plementación de } \\
\text { planta piloto en } \\
\text { investigación e in- } \\
\text { novación de la FAI- } \\
\text { IA de la UNCP El } \\
\text { Mantaro }\end{array}$ & $\begin{array}{l}\text { a). Inadecuada de- } \\
\text { terminación del } \\
\text { Valor Referencial } \\
\text { b) Ampliaciones de } \\
\text { plazo }\end{array}$ & $X$ & $\mathrm{X}$ & $\begin{array}{l}\text { Clores referencia- } \\
\text { les sin sustento } \\
\text { Ejecución de } \\
\text { mayores metrados }\end{array}$ & $\begin{array}{l}\text { Modifica la ruta } \\
\text { crítica de la eje- } \\
\text { cución de obra }\end{array}$ & 3 & 3 & 9 & Alto \\
\hline
\end{tabular}


Tabla 11

Identificación de riesgo, valoración y valor del riesgo

\begin{tabular}{|c|c|c|c|c|c|c|}
\hline \multirow{2}{*}{$\mathbf{N}^{\mathbf{o}}$} & \multirow{2}{*}{ Obra } & \multirow{2}{*}{ Riesgos } & \multicolumn{3}{|c|}{ Valoracion del riesgo } & \multirow{2}{*}{$\begin{array}{l}\text { Valor } \\
\text { Riesgo }\end{array}$} \\
\hline & & & Prob & Imp & Valor & \\
\hline 1 & $\begin{array}{l}\text { Instalación de auditorio de la Universidad Nacio- } \\
\text { nal del Centro del Perú }\end{array}$ & $\begin{array}{l}\text { a) Inadecuada determinación del valor } \\
\text { referencial } \\
\text { b) Ampliaciones de plazo }\end{array}$ & 3 & 3 & 9 & Alta \\
\hline 2 & $\begin{array}{l}\text { Construcción y equipamiento de los laboratorios } \\
\text { de química analítica en la Planta Piloto Metalúr- } \\
\text { gico de Yauris de la Facultad de Metalurgia y de } \\
\text { Materiales de la UNCP. }\end{array}$ & $\begin{array}{l}\text { a) Inadecuada determinación del valor } \\
\text { referencial } \\
\text { b) Ampliaciones de plazo }\end{array}$ & 3 & 3 & 9 & Alta \\
\hline 3 & $\begin{array}{l}\text { Creación de aulas y servicios higiénicos de la } \\
\text { Facultad de Ciencias Agrarias de la UNCP - Rio } \\
\text { Negro Satipo Junín }\end{array}$ & $\begin{array}{l}\text { a) Inadecuada determinación del valor } \\
\text { referencial } \\
\text { b) Ampliaciones de plazo }\end{array}$ & 3 & 2 & 6 & Mediana \\
\hline 4 & $\begin{array}{l}\text { Mejoramiento de los residuos sólidos de la ciu- } \\
\text { dad universitaria de la UNCP. }\end{array}$ & $\begin{array}{l}\text { a) Inadecuada determinación del valor } \\
\text { referencial } \\
\text { b) Ampliaciones de plazo }\end{array}$ & 1 & 3 & 3 & Mediana \\
\hline 5 & $\begin{array}{l}\text { Construcción y equipamiento del pabellón de la- } \\
\text { boratorios de investigación científica y tecnoló- } \\
\text { gica de ciencias agrarias de la UNCP El Mantaro } \\
\text { UNCP Hyo Junín }\end{array}$ & $\begin{array}{l}\text { a) Inadecuada determinación del valor } \\
\text { referencial } \\
\text { b) Ampliaciones de plazo }\end{array}$ & 3 & 2 & 6 & Mediana \\
\hline 6 & $\begin{array}{l}\text { Construcción y equipamiento del pabellón de labo- } \\
\text { ratorios de investigación científica y tecnológica de } \\
\text { las facultades de arquitectura, ingeniería civil, mi- } \\
\text { nas, mecánica, metalúrgica y sistemas de la UNCP }\end{array}$ & $\begin{array}{l}\text { a) Inadecuada determinación del valor } \\
\text { referencial } \\
\text { b) Ampliaciones de plazo }\end{array}$ & 2 & 2 & 4 & Bajo \\
\hline 7 & $\begin{array}{l}\text { Construcción e implementación de planta piloto } \\
\text { en investigación e innovación de la FAIIA de la } \\
\text { UNCP El Mantaro }\end{array}$ & $\begin{array}{l}\text { a) Inadecuada determinación del valor } \\
\text { referencial } \\
\text { b) Ampliaciones de plazo }\end{array}$ & 3 & 3 & 9 & Alto \\
\hline
\end{tabular}

Tabla 12

Valoración del riesgo

\begin{tabular}{ccc}
\hline $\mathbf{N}^{\circ}$ & Obras & Valoración \\
\hline 1 & LP. No 002-2014-CELP & 9 \\
2 & L.P. N $001-2014-C E L P-U N C P$ & 9 \\
3 & AS-SM-2-2016-CSASO-UNCP-1 & 6 \\
4 & OBRAS POR IMPUESTO & 3 \\
5 & L.P. N ${ }^{\circ} 01-2011-U N C P-E O$ & 6 \\
6 & L.P. N N $^{\circ} 001-2010-U N C P-E O$ & 4 \\
7 & AS N N $^{\circ} 01-2017-C O S L P O$ & 9 \\
\hline
\end{tabular}

Figura 1

Valoración del riesgo

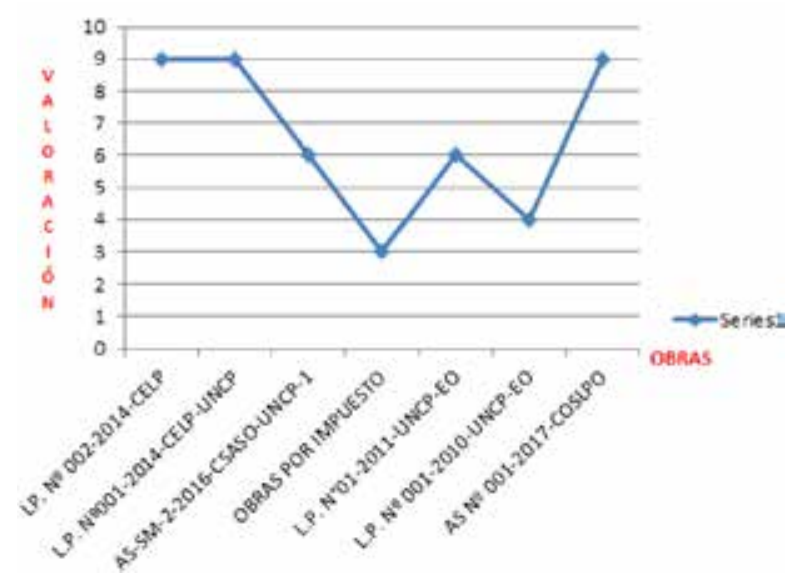

En la fase del proceso de selección, en si conlleva toda actuación, desde difusión de las bases de la licitación pública. Implica la convocatoria, la publicación de bases, inscripción de postores, presentación de consultas y/u observaciones, presentación de propuestas, evaluación de propuestas, determinación de la buena pro y consentimiento de la buena pro e impugnaciones de ser el caso (Según OSCE). Interviniendo los agentes económicos como sujetos de derecho, al inscribirse en el proceso de selección y presentar propuestas para la licitación, son pasibles de ser afectados por una decisión posterior (buena pro) y acciones de impugnación.

Y, en la fase de ejecución de la contratación estatal, conlleva desde el momento de suscribir el contrato hasta la liquidación técnica financiera del mismo. Con ello se clarifica cómo funciona el sistema y donde se presenta los niveles de riesgo como deformación de la contratación pública, al ejecutar una obra pública, dependiendo mucho de como los operadores determinen la modalidad y sistema de contratación, básicamente.

En la Tabla 13 y Figura 2, se tiene que la discusión de resultados sobre determinación del nivel de riesgo, se debe a deficiencias en los planos del proyecto de pre-inversión, debido a un estudio definitivo con carencias técnicas, muchas veces por la falta de especialistas o personal calificado, consecuentemente la poca capacidad de los contratistas en el desempeño de su trabajo. 
Tabla 13

Nivel de riesgo

\begin{tabular}{clc}
\hline $\mathbf{N}^{\circ}$ & \multicolumn{1}{c}{ Obras } & Nivel del riesgo \\
\hline 1 & LP. $\mathrm{N}^{\circ}$ 002-2014-CELP & Alta \\
2 & L.P. No001-2014-CELP-UNCP & Alta \\
3 & AS-SM-2-2016-CSASO-UNCP-1 & Medio \\
4 & OBRAS POR IMPUESTO & Medio \\
5 & L.P. No01-2011-UNCP-EO & Medio \\
6 & L.P. No 001-2010-UNCP-EO & Bajo \\
7 & AS N N $^{\circ} 001-2017-C O S L P O-$ & Alto \\
\hline
\end{tabular}

Figura 2

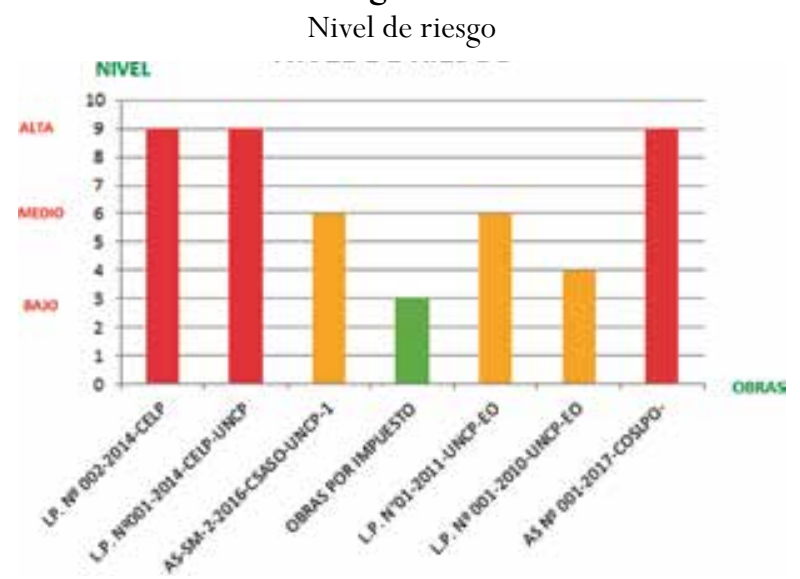

\section{Conclusiones}

- En la investigación sobre evaluación del nivel de riesgo en las contrataciones para ejecución de obras, se identificó una serie de factores; entre ellas, la modalidad y tipo de convocatoria no son las adecuadas. A ello se suma que, los estudios definitivos son deficientes y con una serie de debilidades, que conlleva a la asignación de recursos económicos adicionales y culminación en forma inoportuna.

- Todos los proyectos de inversión evaluados y la ejecución se realizaron bajo la modalidad de suma alzada, cuya característica es que deberían de culminar de acuerdo al expediente técnico, bases y propuesta del contratista, pero al verificar las liquidaciones técnicas y financieras que cierra el expediente de contratación, se identificó adicionales de obras con sus respectivas valorizaciones, ampliaciones de plazo y, en general, sobrecostos que generan perjuicio económico al Estado.

- También es preocupante, cuando se identificó pagos a los contratistas por concepto de multas por pagos retrasados de las valorizaciones a los contratistas; además, de las diferencias por efecto de la fórmula polinómica, diferencias del IGV, entre otras. Una debilidad de gestión administrativa con riesgo alto, el cual conlleva a determinación de responsabilidad, el cual generó perjuicio económico a la entidad y al Estado; curiosamente incluso se viene ventilando algunas conciliaciones como solución de controversia.

- Sobre el personal técnico, operadores logísticos, miembros del comité y área técnica - usuaria reflejan conocimientos escasos y limitados en administración de riesgos para la ejecución de los PIPs, al cual, sumado las malas prácticas, vacíos en el sistema normativo de las contrataciones publica, conlleva a un riesgo alto, ello se refleja en obras inconclusas, con problemas (conciliación o arbitraje), siendo una puerta abierta para que los contratistas manejen a su antojo los contratos suscritos.

\section{Recomendaciones}

- Se recomienda mejorar la selección de los técnicos consultores, que diseñan los estudios preliminares y definitivos de los PIPs. La deficiente formulación conlleva a la deformación dentro de su ejecución, distorsionando plazos e inoportunas, paralizaciones, crean adicionales. Con una adecuada administración de riesgos, se evitarían la generación de deformaciones y problemas.

- Al interior de la universidad, se debe implementar lineamientos para la gestión de riesgos con instrumentos de control descritos en la presente investigación, para que ello conlleve a un adecuado manejo racional, prudente y eficiente de los escasos recursos que se asigna a la universidad, toda vez que existe cantidad de necesidades por atender a la comunidad universitaria.

- También, se recomienda mejorar al interior de la universidad la cultura organizacional del personal administrativo y docente, existe bastante apatía, que conlleva a niveles de irresponsabilidad y desidia, reflejandose la cantidad de problemas, de carácter legal y un inadecuado sistema de solución de controversias donde las empresas son ganadoras.

- Habiéndose determinado que no existen instrumentos que permitan mediar la calidad del gasto público en la universidad, se hace necesario implementar lineamientos que conlleven a efectuar el uso adecuado de los recursos con políticas de preservación y el uso racional y adecuado de los bienes del Estado. 


\section{Referencias bibliográficas}

Álvarez Pedroza, A. (2010). Comentarios a la Ley y Reglamento de Contrataciones del Estado. Lima: Marketing Consultores. Escuela de Gerencia Gubernamental.

Contraloría General de la República. Resolución 196 2010-CG y su Directiva No. 002-2010-CG/OEA sobre aprobación de adicionales de obra. En www. contraloria.gob.pe.

Contratando con el Estado. Blog sobre contratación estatal. En http://contratandoconelestado. blogspot.com/

Derecho público y arbitraje. Blog de Derik Latorre Boza. En: http://blog.pucp.edu.pe/blog/derechopublicoyarbitraje

Instituto de la Construcción y Gerencia. Libros, artículos, videos, cursos, sobre contratación estatal y otros. En www.construccion.org.pe

Linares Jara, M. y Martín Tirado, R. (2007). Contrataciones y adquisiciones del Estado. Lima: Editora Jurídica Grijley E.I.R.L.

Linares Jara, M. (2002). El contrato estatal. Lima: Editora Jurídica Grijley E.I.R.L. (s.a.) Normas Legales de Construcción (2010). Lima: Fondo Editorial del Instituto de Construcción y Gerencia.

Organismo Supervisor de las Contrataciones del Estado. Ley de Contrataciones y su Reglamento, opiniones, pronunciamientos, directivas, comunicados, resoluciones del tribunal, etc. En www. osce.gob.pe

Portal de Contrataciones del Estado. Artículos y capacitación sobre la contratación estatal. En www.perucontrata.com.pe

Salinas Seminario, M. (2011). Supervisión de obras. Lima: Fondo Editorial del Instituto de Construcción y Gerencia.

Salinas Seminario, M. (2009). Liquidación técnico financiera de obras públicas. Lima: Fondo Editorial del Instituto Pacifico SAC.

Salinas Seminario, M. (2009). Administración de contratos de obras. Lima: Fondo Editorial del Instituto de Construcción y Gerencia.

Salinas Seminario, M. (2009). Elaboración de expedientes técnicos para obras. Lima: Fondo Editorial del Instituto de Construcción y Gerencia. 